\title{
BOARD OF DIRECTORS
}

\section{FFICERS}

onorary President resident ast President

irst Vice-President

econd Vice-President

reasurer

orresponding Secrelary

ecording Secretary

\section{PPOINTED DIRECTORS}

lue Jay Editor

lue Jay Books/hop

irculatuon

onservation

indangered Sperie's

irasslands Park

coal Socicties

embership

wisletter Editors

pecial Publications

Elizabeth Cruickshank 2339 Athol St., Regina, S4T 3 G4 Gary W. Scib

Margaret Beleher

Ole Nielsen

Frank Switzer

George R. Dodd

Gary Wobeser

Dale Hjertaas

2.210414 th Ave., Regina, S4P 0X6

26(0) Winnipeg St., Regina, S4P IH8

327 Poplar Cresc., Saskatoon, S7M 0A8

1301 Shannon Rd., Regina, S4S 5K9

33 Malone Cresc., Regina, S4S 5RI

209 Tucker Cresc., Saskatoon, S7H 3J 1 1317 Osler St., Saskatoon, S7N (oV!

J. Bernard Gollop

Frank Brazier

2202 York Ave., Saskatoon, S7J IJ I

2657 Cameron St., Regina, S4T 2W5

Lorne Seott

Saskatchewan Museum of Natural History, Regina Frank Switzer

1301 Shannon Rd., Regina, S4S 5K9 James R. Jowsey 2635 Nineteenth Ave., Regina, S4T I X2 George F. ledingham 2335 Athol St., Regina, S4T 3 G4 Lorne Seott

Saskatchewan Museum of Natural History, Regina Lynn Oliphant

\section{Saskatchewan Cres. W.} Saskatoon, S7M 0A4

Lloyd O. T. Peterson

Wm. and Joyce Anaka Box 866, Indian Head, SOG 2KO

Box 211. Yorkton, S3N 2V8 C. Stuart Houston 863 University Dr., Saskatoon, S7N 0J8

\section{EPRESENTATIVES AT LARGE}

Ron E. Andrews

843 Athabasca St. W., Moose Jaw, Sask. S6H 2E2 73 Cardinal Cresc. Regina, Sask. S4S 4 Y6

jeorge Ferguson Broadview, Sask. SOG OKO ooug Francis Nokomis, Sask. SOG 3 R0

im Hamilton , Raymore, Sask. S0A 3J0

Nayne Harris

Box 93, Raymore, Sask. S0A 3J0

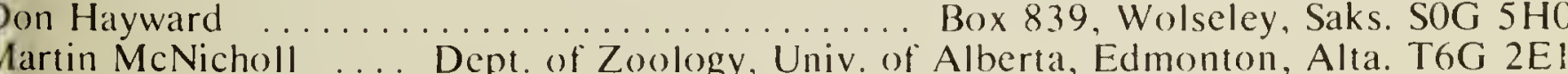

Christine Pike ...................... Box 117, Waseca, Sask. SOM 3A0

tan Riome

Box 2103, Nipawin, Sask. SOE 1 E0 aul Rump ...................... 3320 Angus St., Regina, Sask. S4S IP8 pencer Sealy ....... Dept. of Zoology, Univ. of Manitoba, Winnipeg, Man. R3T 202 Jary Wobeser .................. 209 Tucker Cresc., Saskatoon, Sask. S7H 3JI

\section{PRESIDENTS OF LOCAL SOCIETIES}

Gort Qu'Appelle ........ Bernard de Vries

ndian Head ........... Mary Skinner

Maple Creek ............ D. Bromley

Joose Jaw ............. Patricia Kern

Regina ............. W. Lloyd Hipperson

askatoon ........... Don McRobbie

swift Current .......... Jan Looman

Porkton ............. Phil Pawluck

Jniversity Biology Clubs . Richard Collins

Dale Botting
Box 1043, SOG 1 SO

P.O. Box 777. SOG $2 \mathrm{KO}$ SON INO

1053 Chestnut Ave., S6H 1 A7 3708 Albert St.. S4S 3P9 525 - 4th Ave. N., S7K 2 M 5 491 - 2nd Ave. S.E.. S9H 3J7

163 Peaker Ave., S3N IS6

University of Regina, S4S 0A2 University of Saskatchewan, S7N OW0 